\title{
Multiple points of view of heteronuclear NOE: long range vs short range contacts in pyrrolidinium based ionic liquids in the presence of Li salts. ${ }^{\S}$
}

Franca Castiglione ${ }^{1}$, Giovanni Battista Appetecchi ${ }^{2}$, Stefano Passerini ${ }^{3,4}$, Walter Panzeri ${ }^{5}$, Serena Indelicato ${ }^{6}$, Andrea Mele ${ }^{1,5^{*}}$

Department of Chemistry, Materials and Chemical Engineering "G. Natta", Politecnico di Milano, Piazza L. Da Vinci 32, 20133 Milano, Italy

${ }^{2}$ ENEA, Agency New Technology, Energy and Sustainable Economic Development, UTRINN IFC, Via Anguillarese 301, I-00123 Rome, Italy

${ }^{3}$ Helmholtz Institute UIm (HIU), Electrochemistry I, Helmholtz Strasse 11, 89081 Ulm, Germany

${ }^{4}$ Karlsruhe Institute of Technology, P.O. Box 3640, 76021 Karlsruhe, Germany.

${ }^{5}$ CNR - Istituto di Chimica per il Riconoscimento Molecolare, Via L. Mancinelli, 7, 20131 Milano, Italy

${ }^{6}$ Department STEBICEF, University of Palermo, Via Archirafi n.32, I-90123, Palermo, Italy

*Email: andrea.mele@polimi.it

$\S$ Dedicated to the memory of Alessandro Bagno (University of Padova) passed on March 23, 2015.

Abstract

The nuclear Overhauser enhancement (NOE) is a powerful tool of NMR Spectroscopy extensively used to gain structural information in ionic liquids (ILS). A general model for the distance dependence of intermolecular NOE in ILS was recently proposed showing that NOE spots beyond the first solvation shell and accounts for long-range effects. This conclusion prompted for a deep rethinking of the NOE data interpretation in ILs. In this paper we present an extensive and quantitative study of $\mathrm{N}$-propyl- $\mathrm{N}$-methyl pyrrolidinium bis(trifluoromethanesulfonyl)imide ( $\mathrm{PYR}_{13} \mathrm{TFSI}$ ), the homologue with bis(fluorosulfonyl)imide (PYR ${ }_{13} \mathrm{FSI}$ ), and their mixtures with LiTFSI based on $\left\{{ }^{1} \mathrm{H}-{ }^{19} \mathrm{~F}\right\}$ and $\left\{{ }^{1} \mathrm{H}-{ }^{7} \mathrm{Li}\right\} \mathrm{NOE}$ correlation experiments (HOESY). The former is mainly tuned on long-range interactions, the latter on short-range ones, due to the small and large Larmor frequency differences of the involved nuclei. The collected data are discussed in two different way: long-range $\left\{{ }^{1} \mathrm{H}-{ }^{19} \mathrm{~F}\right\}$ NOEs spot on the polar/apolar domains within the ILs, whereas shortrange (e.g. regarding the first coordination shell) $\left\{{ }^{1} \mathrm{H}-{ }^{7} \mathrm{Li}\right\} N O E s$ describe the contacts between first neighbors, with interesting correlation with the distances' statistics derived by crystallographic data of related systems.

\section{Introduction}

The unique properties of ionic liquids (ILS) and the growing scientific and technological interest they raise prompt for a thorough understanding of their structure at increasing levels of details. In the large repertoire of physical methods used for ILs' characterization, NMR spectroscopy is still playing an important role, outlined in some recent review articles [1, 2]. In turn, the nuclear Overhauser enhancement (NOE) is one of the most powerful NMR tool for the assessment of three dimensional structure of molecules [3] and for the detection of intermolecular interactions [4]. The first application of NOE to ILs dated back 1995 by the group of Osteyoung [5]. Since then, many applications of NOE for ILs have been published, either homonuclear (typically $\left\{{ }^{1} \mathrm{H}-{ }^{1} \mathrm{H}\right\} \mathrm{NOE}$ ) [6-9] or heteronuclear (most of the cases $\left\{{ }^{1} \mathrm{H}-{ }^{19} \mathrm{~F}\right\} \mathrm{NOE}$ ) [10-12]. The main strength of the NOE approach is that it is originated by the mutual relaxation (cross-relaxation) of NMR interacting nuclei close in space, thus providing precious information on the three dimensional structure of molecules and/or aggregate containing the interacting nuclei. The NOE stems from the coupling of the magnetic moments of the interacting spins, generally indicated as $I$ and $S$. The magnetic dipole-dipole interaction fluctuates with time, due to molecular motion. Such fluctuations are the physical foundation for magnetization transfer and cross relaxation, indicated with $\sigma_{I s}$. More formally, the spectral density function $J(\omega)$ describes the modulation of the magnetic dipole-dipole interactions due to the molecular motion. In the case of intramolecular NOE, the distance $r_{1 S}$ between $I$ and $S$ is constant (if we 
neglect segmental internal motions ) and, therefore, the rotational reorientation of the molecule is the main source of dipole-dipole fluctuations affording the cross-relaxation. The most popular consequence of such mechanism is in the $r^{-6}$ distance dependence of the cross-relaxation rate, leading to the "rule of thumb" of vanishing NOE at $r_{\text {IS }}>5 \AA$. Thus, intramolecular NOE spots on short-range interactions, mainly.

A formal theory for the intermolecular NOE is complicated by I and $S$ being placed on different molecules undergoing both rotational and translational rearrangements. Different models have been developed in order to account for these factors [13-14], all leading to the conclusion that the intermolecular NOE extends beyond the $5 \AA$ threshold, thus assuming a long-range, rather than short-range, character. In the context of ILs, these findings have a quite important fall-out, as many investigations on the local structure of ILs are based on the intermolecular homo- [6-9] and heteronuclear NOE [10,12]. As remarked, many ILs are composed of cations containing $\mathrm{H}$ atoms in an organic frame (e.g. imidazolium, pyridinium, ammonium, pyrrolidinium, etc), and anions that very often do contain $F$ atoms instead of $\mathrm{H}$ atoms, typically sulfonylimide-based anions. In such cases, the homonuclear NOE correlation experiments currently available on modern spectrometers, namely $\left\{{ }^{1} \mathrm{H}-{ }^{1} \mathrm{H}\right\} \mathrm{NOESY}$ or ROESY and $\left\{{ }^{19} \mathrm{~F}-{ }^{19} \mathrm{~F}\right\} \mathrm{NOESY}$, provide details on the cation-cation and anion-anion organization, respectively, while the heteronuclear $\left\{{ }^{1} \mathrm{H}-{ }^{19} \mathrm{~F}\right\} \mathrm{HOESY}$ measurements spot on the cation-anion pattern. Here the acronyms NOESY and ROESY refer, respectively, to two dimensional, homonuclear NOE correlation experiments in the lab frame and in the rotating frame, in the order. HOESY refers to two dimensional heteronuclear NOE correlation experiments. [15]

Recently, Gabl, Steinhauser and Weingärtner (GSW) proposed a mathematical model for the prediction of $J(\omega)$ in the case of intermolecular cross-relaxation in ILs $[16,17]$. The new concept introduced by the GSW approach is that the distance law of intermolecular NOE strongly depends on the Larmor frequencies of the interacting nuclei $I$ and $S, \omega_{l}$ and $\omega_{s}$, respectively. The $r^{-6}$ short-range dependence, strictly true for the intramolecular cross-relaxation, turns into a more general $r^{-n}$ distance law, with $\mathrm{n}<6$. Consequently, longrange effects can be detected in intermolecular NOE experiments whenever the frequencies of the crossrelaxing nuclei are close enough, as in the homonuclear case $\left\{{ }^{1} \mathrm{H}-{ }^{1} \mathrm{H}\right\}$, or in heteronuclear cases such as $\left\{{ }^{1} \mathrm{H}-{ }^{19} \mathrm{~F}\right\}$. In particular, the authors estimated $\mathrm{n} \approx 2.5$ for $\left\{{ }^{1} \mathrm{H}^{19} \mathrm{~F}\right\}$ and a value for the exponent $\mathrm{n}$ even smaller for the homonuclear case. As we anticipated earlier [18], the advancement of the intermolecular NOE theory calls for a re-thinking of the current criteria for the interpretation of intermolecular NOE in ILs.

In this paper we present a collection of heteronuclear NOE experiments on a simple model amenable of interpretation at the light of the new theoretical advancements. It is intended to be a case-study where both short and long range effects can be considered and discussed as a paradigm of the up-to-date method of NOE data interpretation. Thus, the main purpose of this work is to promote a debate on the use and interpretation of heteronuclear, intermolecular NOEs in assessing the local order of ILs, rather than providing (or not) a validation of the GSW model.

In order to reach this goal, the following points have been considered: we have chosen two ILs based on the $N$-propyl- $N$-methyl pyrrolidinium cation $\left(\mathrm{PYR}_{13}\right)$ combined with two different perfluorinated anions. The two anions are bis(trifluoromethanesulfonyl)imide (TFSI) and bis(fluorosulfonyl)imide (FSI), in the order. The molecular formulae and the atom labels are depicted in Figure 1. All the systems were examined as pure liquids and as mixture with LiTFSI in the molar composition 0.9 PYR $_{13} \mathrm{X}-0.1$ LiTFSI (X= TFSI, FSI). A single case of a ternary mixture (0.3 PYR13TFSI - 0.6 PYR13FSI - 0.1 LiTFSI) is also reported as supporting example for the assessment of Li molecular environment. It is important to remind the technological importance of these systems as innovative electrolyte components for safer lithium ion battery systems [19]. Since their unique properties, e.g., non-flammability, negligible vapor pressure, remarkable ionic conductivity, high thermal, chemical and electrochemical stability, low heat capacity, ability to dissolve inorganic (including lithium salts), organic and polymeric materials [20-23], ILs have attracted growing attention for replacing organic compounds currently used not only in lithium batteries [19], but also in a wide variety of electrochemical devices [24-30], resulting in improved safety in case of overheating/overcharging which can lead to flaming/explosion. It is to note that safety issues have been preventing till now the wide diffusion of lithium batteries in large scale applications such as automotive, electric storage from renewable power sources, smart grids, etc. In addition, the possibility to widely 
combine a large variety of cations and anions gives the freedom to design the most suitable ionic liquids for matching the requirements of the electrochemical device. Viable lithium battery ionic liquids [19] are formed by alkylimidazolium, saturated alkyl quaternary ammonium [cyclic (pyrrolidinium, piperidinium) or acyclic (tetraalkylammonium)] cations in combination with hydrophobic perfluoroalkylsulfonylimide anions. The mole fraction of LiTFSI, e.g., 0.1, was chosen because it confers from good to high $\mathrm{Li}^{+}$conduction values to electrolytes and, at the same time, falls rather far from the solid eutectic composition [31] (e.g., which might take place upon high current rates conditions, depleting the battery performance).

From the NMR point of view, and in the economy of the present paper, these systems allowed us to carry out two different types of HOESY experiments: $\left\{{ }^{1} \mathrm{H}^{19} \mathrm{~F}\right\}$ and $\left\{{ }^{1} \mathrm{H}-{ }^{7} \mathrm{Li}\right\}$. The former case, previously discussed, is expected to reflect the ions organization also beyond the first solvation shell. Conversely, in the latter case, very rarely encountered in the recent literature on ILs [18,32-34], the Larmor frequency of the two nuclei, ${ }^{1} \mathrm{H}$ and ${ }^{7} \mathrm{Li}$, are significantly far apart, thus smoothing, if not completely eliminating, the long-range component to the cross-relaxation, and leaving the short-range, $r^{-6}$ mediated distance dependence in the intermolecular NOE. The combined use of $\left\{{ }^{1} \mathrm{H}-{ }^{19} \mathrm{~F}\right\} \mathrm{HOESY}$ and $\left\{{ }^{1} \mathrm{H}-{ }^{7} \mathrm{Li}\right\} \mathrm{HOESY}$ is thus expected to provide an exhaustive structural picture, comprising both long and short range structural motives. The theoretical foundation of this statement is briefly outlined in the Materials and Methods section, as "Intermolecular NOE and interacting nuclei distance: some key concepts".

$"$.

a)

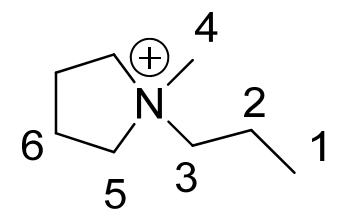

b)<smiles>[CH2-]S[Os]N[Os]OC(F)(F)F</smiles>

c)

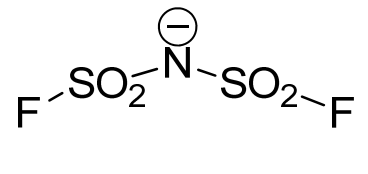

Figure 1. Chemical structures and atom numbering of the cation (a) and the anions TFSI (b) and FSI (c).

\section{Materials and Methods}

$\underline{\text { Ionic Liquids Synthesis }}$

The PYR 13 TFSI and $\mathrm{PYR}_{13} \mathrm{FSI}$ ionic liquids were synthesized through a procedure developed at ENEA and described in details elsewhere [35]. The chemicals, namely $N$-methylpyrrolidine (98 wt.\%) and 1bromopropane (99 wt.\%) and the sorbents, e.g., activated carbon (Darco-G60) and alumina (acidic), were purchased by Aldrich. Lithium bis(trifluoromethanesulfonyl)imide, LiTFSI (> 99.9 wt.\%), and sodium bis(fluorosulfonyl)imide, NaFSI (> 99.9 wt.\%), were provided by $3 \mathrm{M}$ and Solvionic, respectively. Deionized $\mathrm{H}_{2} \mathrm{O}$ was obtained with a Millipore ion-exchange resin deionizer.

The $\mathrm{N}$-methyl- $\mathrm{N}$-propylpyrrolidinium bromide $\left(\mathrm{PYR}_{13} \mathrm{Br}\right)$ precursor was synthesized by reacting $\mathrm{N}$ methylpyrrolidine, previously dissolved in deionized water, with the appropriate amount of 1bromopropane. After $30-60$ minutes stirring at $70^{\circ} \mathrm{C}$, a transparent, yellowish, aqueous solution of $\mathrm{PYR}_{13} \mathrm{Br}$ was obtained. The precursor was then purified through activated carbon and acidic alumina. The liquid fraction (a clear, colorless, highly concentrated solution of $\mathrm{PYR}_{13} \mathrm{Br}$ in water) was separated from the solid one (sorbent materials) by vacuum filtering and separately collected. Successively, the solid phase was rinsed with deionized water to recover the precursor trapped through the sorbent materials, obtaining a further $\mathrm{PYR}_{13} \mathrm{Br}$ aqueous solution (clear and colorless), which was unified to the previous one. 
formation of hydrophobic $\mathrm{PYR}_{13} \mathrm{TFSI}$ (or $\mathrm{PYR}_{13} \mathrm{FSI}$ ) and hydrophilic $\mathrm{LiBr}$ (or $\mathrm{NaBr}$ ). After 10-15 minutes, the mixing was interrupted, allowing phase separation to occur. The upper phase was mostly composed of water, $\mathrm{LiBr}$ (or $\mathrm{NaBr}$ ) and LiTFSI (or NaFSI) excess whereas the lower one was constituted of $\mathrm{PYR}_{13} \mathrm{TFSI}$ (or $\mathrm{PYR}_{13} \mathrm{FSI}$ ) with traces of water and $\mathrm{LiBr}$ and LiTFSI (or $\mathrm{NaBr}$ and $\mathrm{NaFSI}$ ). After removal of the aqueous phase, the ionic liquids were rinsed several times with deionized water to remove water-soluble salts ( $\mathrm{LiBr}$ or $\mathrm{NaBr}$ and excess of LiTFSI or NaFSI) and other impurities. Successively, the ionic liquids were placed in a vacuum rotary evaporator at $90^{\circ} \mathrm{C}$ to remove the most water and, finally, were dried under high vacuum $\left(10^{-5}\right.$ $\mathrm{mmHg}$ by a turbo-molecular pump) at $20^{\circ} \mathrm{C}$ for two hours, followed by two hours at $60^{\circ} \mathrm{C}$ and at least eighteen hours at $120^{\circ} \mathrm{C}$. Finally, the dry ionic liquids were stored in sealed glass tubes. The vacuum drying and sealing steps were performed inside the dry room (R.H. $<0.1 \%)$.

The procedure route allowed obtaining transparent, colorless, odorless ionic liquid materials with a moisture content below 2 ppm.

PYR $_{13}$ TFSI-LiTFSI and PYR ${ }_{13}$ FSI-LiTFSI electrolyte mixtures were prepared (inside the dry room) by stirring the components, blended in the proper stoichiometric amounts, for $5-10$ minutes at $50^{\circ} \mathrm{C}$.

\section{NMR Spectroscopy}

The ${ }^{1} \mathrm{H}$ and ${ }^{19} \mathrm{~F}$ NMR spectra were recorded on a Bruker Avance 500 spectrometer operating at the 500.13 $\mathrm{MHz}$ proton frequency equipped with a QNP four nuclei switchable probe. The ${ }^{7} \mathrm{Li}$ spectra were acquired on a Bruker DRX 500 spectrometer equipped with a broadband probe. The IL samples (0.5-1.0 g each) were transferred in a $5 \mathrm{~mm}$ NMR tube inside the dry-room to avoid any water contamination. The NMR tubes, equipped with a co-axial capillary containing d6-DMSO as internal chemical shift reference, were immediately flame-sealed after loading the sample. Heteronuclear $\left\{{ }^{1} \mathrm{H}_{-}{ }^{19} \mathrm{~F}\right\}$ and $\left\{{ }^{1} \mathrm{H}-{ }^{7} \mathrm{Li}\right\} \mathrm{HOESY}$ experiments were acquired using the inverse-detected pulse sequence proposed by Alam et al. [36] with 128 increments in the $\mathrm{t} 1$ dimension and sixteen scans for each experiment. The range for the mixing time was of $20 \mathrm{~ms}-4 \mathrm{~s}$ for the $\left\{{ }^{1} \mathrm{H}-{ }^{19} \mathrm{~F}\right\}$ experiments and $50 \mathrm{~ms}-3 \mathrm{~s}$ for the $\left\{{ }^{1} \mathrm{H}-{ }^{7} \mathrm{Li}\right\}$ experiments. The sample PYR ${ }_{13} \mathrm{FSI}-\mathrm{LiTFSI}$ contains both the FSI and TFSI anions with a fluorine shift difference of about $133 \mathrm{ppm}$ at $305 \mathrm{~K}$ (with the TFSI signal observed at higher field). For this sample two separate $\left\{{ }^{1} \mathrm{H}-{ }^{19} \mathrm{~F}\right\}$ HOESY experiments have been performed using a Gaussian $90^{\circ}$ soft pulse centered on the TFSI and FSI frequency respectively in each experiment. The $2 \mathrm{D}$ data sets were processed with $\mathrm{SI} 1=2 \mathrm{~K}$ points and $\mathrm{SI} 2=512$ points. Squared cosine bell window multiplication was then applied in both dimensions before 2D Fourier transform. The cross-peak integrals were measured using the Bruker software. The peak volume of each cross-peak has been corrected by considering the contribution of the number of spins. The cross-peak intensity arising from $N_{1}$ equivalent spins I $\left({ }^{1} \mathrm{H}\right)$ and $\mathrm{N}_{S}$ equivalent spin $\mathrm{S}\left({ }^{19} \mathrm{~F}\right.$ or $\left.{ }^{7} \mathrm{Li}\right)$ is proportional to the ratio of $\mathrm{N}_{1} \mathrm{~N}_{S} /\left(\mathrm{N}_{1}+\mathrm{N}_{S}\right)$; [37] therefore, for semi-quantitative comparison, the original peak volume has been corrected by dividing the original integral by the value $\mathrm{N}_{1} \mathrm{~N}_{\mathrm{S}} /\left(\mathrm{N}_{1}+\mathrm{N}_{\mathrm{S}}\right)$. For all the experiments, the temperature was set and controlled at 305 K.

\section{$\underline{\text { Intermolecular NOE and interacting nuclei distance: some key concepts }}$}

A summary of NOE theory is far beyond the scope of this work; the reader interested in the development of the theory and applications can refer to specialized textbooks [3] The purpose of this paragraph is to highlight only few concepts useful for the discussion of intermolecular NOEs and their dependence upon the distance $r$ between the interacting nuclei. The usual notation of $I$ and $S$ for the different nuclear species close in space and able to undergo cross-relaxation, thus to develop heteronuclear NOE, is here adopted. No attempt to demonstrate equation or to derive them is reported here, as extensively treated in references 16 and 17 .

In an heteronuclear NOE experiment, the cross-relaxation rate $\sigma_{I S}$. is given by the equation (1):

$\sigma_{l s}=0.9 \mathrm{~J}\left(\omega_{1 .}+\omega_{s}\right)-0.1 \mathrm{~J}\left(\omega_{1 .}-\omega_{s}\right)$ 
where $\omega_{I}$ and $\omega_{S}$ are the Larmor frequencies of the two interacting spins $I$ and $S$. The term $J\left(\omega_{1 .}+\omega_{S}\right)$ is the spectral density function at the sum frequency $\omega_{4}=\left(\omega_{1}+\omega_{S}\right)$ and the term $J\left(\omega_{1}--\omega_{S}\right)$ is the spectral density at the difference frequency $\omega_{2}=\left(\omega_{1}-\omega_{S}\right)$. The term $\sigma_{I S}$ is essentially a linear combination of two terms $J\left(\omega_{4}\right)$, accounting for the short range interactions, and $J\left(\omega_{2}\right)$, giving the long range contribution. Therefore, the value of $\sigma_{I S}$ strongly depends on the value of $\omega_{4}$ and $\omega_{2}$, that means how far the $S$ nucleus resonates from the I spins (usually protons). The recent GSW theory on heteronuclear NOE is based on a more complex expression for the spectral density function, which considers the dipole-dipole interactions modulated by translational and rotational motions of the ions and ion diffusion. This theory was applied to ILs and extended the $r^{-6}$ short-range dependence in a more general $r^{-n}$ law, with $1<\mathrm{n}<6$. Their calculations are focused on the $\left\{{ }^{1} \mathrm{H}^{-19} \mathrm{~F}\right\}$ NOEs, that means $\omega_{2}=0.16 \mathrm{GHz}$ and $\omega_{4}=4.85 \mathrm{GHz}$ for a $400 \mathrm{MHz}(2.51 \mathrm{GHz})$ spectrometer. The difference in the resonance frequency between proton and fluorine is very small, consequently the difference frequency $\omega_{2}$ is more than one order of magnitude smaller than the sum frequency $\omega_{4}$. The authors demonstrated that the long-range contribution is dominant in highly viscous media like ILs despite the fact that it is present in eq. (1) with a numerical coefficient six times smaller than the corresponding for the sum of frequency. The results are different when we consider $\left\{{ }^{1} \mathrm{H}-{ }^{7} \mathrm{Li}\right\} \mathrm{NOEs}$. In this case $\omega_{2}=1.53 \mathrm{GHz}$ and $\omega_{4}=3.48 \mathrm{GHz}$, thus the two frequencies are close enough and both account for the short-range interactions with $\mathrm{n} \approx 6$.

\section{Results and Discussion}

In this study we have considered two pure ionic liquids and two mixtures with LiTFSI. For clarity, the samples are indicated according to the composition in molecules normalized to the arbitrary value 100 . The code is summarized in Table 1.

Table 1. Composition (molecules) of the examined systems.

\begin{tabular}{|c|c|c|c|c|c|}
\hline \multirow[t]{2}{*}{ Sample } & \multicolumn{4}{|c|}{ Composition (in molecules) } & \multirow[t]{2}{*}{ code } \\
\hline & $\mathrm{PYR}_{13}$ & TFSI & FSI & $\mathrm{Li}$ & \\
\hline $\mathrm{PYR}_{13} \mathrm{TFSI}$ & 100 & 100 & 0 & 0 & PY1100 \\
\hline 0.9 PYR $_{13}$ TFSI + 0.1 LiTFSI & 90 & 100 & 0 & 10 & PY9101 \\
\hline $\mathrm{PYR}_{13} \mathrm{FSI}$ & 100 & 0 & 100 & 0 & PY1010 \\
\hline $0.9 \mathrm{PYR}_{13} \mathrm{FSI}+0.1 \mathrm{LiTFSI}$ & 90 & 10 & 90 & 10 & PY9191 \\
\hline 0.3 PYR $_{13}$ TFSI - 0.6PYR 13 FSI - 0.1 LiTFSI & 90 & 40 & 60 & 10 & PY9461 \\
\hline
\end{tabular}

\section{Fluorine-proton HOESY}

NMR heteronuclear $\left\{{ }^{1} \mathrm{H}_{-}^{19} \mathrm{~F}\right\}$ NOE correlation experiments were used to gain information on cation-anion interactions and aggregation motives in the pure IL (PYR $\left.{ }_{13} T F S I\right)$. In the second part of this section the effect of IL doping with LiTFSI is monitored by heteronuclear NOE and discussed in terms of structural changes induced by the presence of the Li salt.

The 2D plot of the experiment performed on pure PYR ${ }_{13}$ TFSI shows the dipolar contacts among selected protons of the cation and the fluorine nuclei of the TFSI anion. The contour map of Figure 2 is intended to be a snapshot of the dipolar contacts among the $\mathrm{F}$ nuclei of the anion with the collection of $\mathrm{H}$ nuclei of the cation. 


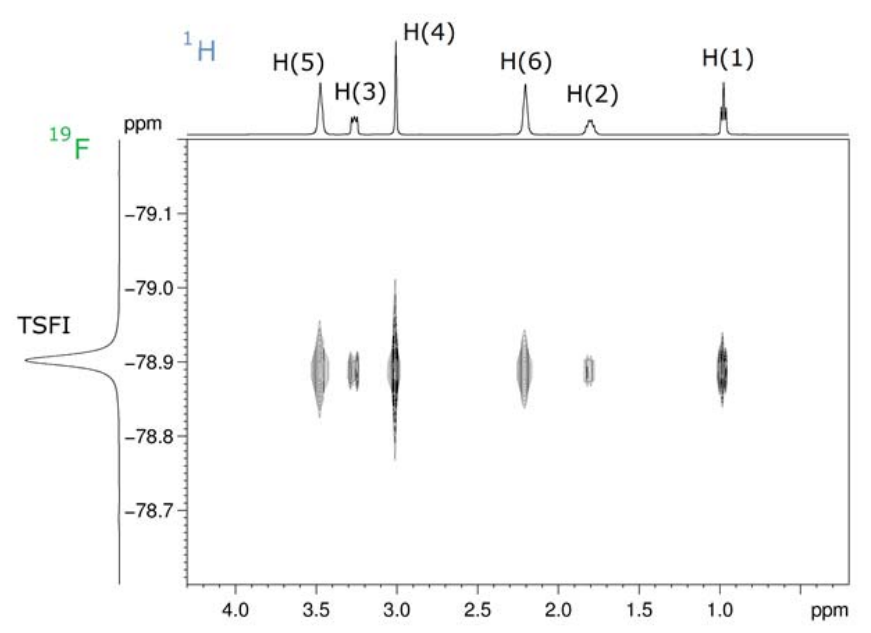

Figure 2. Contour plot of $\left\{{ }^{1} \mathrm{H}-{ }^{19} \mathrm{~F}\right\} \mathrm{HOESY}$ experiment on pure PYR ${ }_{13} \mathrm{TFSI}$ with ${ }^{1} \mathrm{H}$ spectrum (top) and ${ }^{19} \mathrm{~F}$ spectrum (left) acquired with a mixing time of $50 \mathrm{~ms}$. The ${ }^{1} \mathrm{H}$ NMR spectrum assignment is given on top of each peak. The ${ }^{19} \mathrm{~F}$ NMR spectrum consists of a single resonance.

In order to derive more quantitative information on the spatial relationship of anions and cations, the kinetics of NOE build-up was studied by performing an array of HOESY at different mixing time for the magnetization transfer via cross-relaxation. More details on the theory can be found in the literature [12] and will not be repeated here. Suffice to mention here the fact that we choose the so-called initial rate approximation to obtain the cross-relaxation rates $\sigma_{I S}$, in turn related to the selected proton (on the cation) -fluorine (on the anion) internuclear distance $\left(r_{i s}\right)$. The results are shown in Figure 3 as full plot of the normalized cross-peak intensity vs mixing time for PYR ${ }_{13}$ TFSI and its mixture with LiTFSI. The same protocol was applied in the case of pure $\mathrm{PYR}_{13} \mathrm{FSI}$ (curve not shown) and for the mixtures with LiTFSI. In the latter case (sample PY9191), the ${ }^{19} \mathrm{~F}$ NMR spectrum contained two distinct singlets due to the two different anions (TFSI and FSI). The observed chemical shift difference, $\Delta \delta \approx 133 \mathrm{ppm}$, made troublesome the use of the standard pulse sequence employed for the samples containing TSFI only. Therefore, the $\left\{{ }^{1} \mathrm{H}-{ }^{19} \mathrm{~F}\right\} \mathrm{NOE}$ build-up curves were obtained in two separate experiments carried out with a band selective pulse sequence able to extract the NOE contacts of TFSI and FSI. In all the cases, the build-up curves show a linear part at low mixing times, usually in the $0-300 \mathrm{~ms}$ range. However, in order to avoid over-interpretation of the data and errors due to neglecting the different relaxation rates of the nuclei [12], we restricted the linear region to the 0-150 ms range and used only those data for the linear regression. As an example, the linear plots from data of Figure 3 are shown in Figure 4. The slope of the regression curves is proportional to $\sigma_{l s}$. 

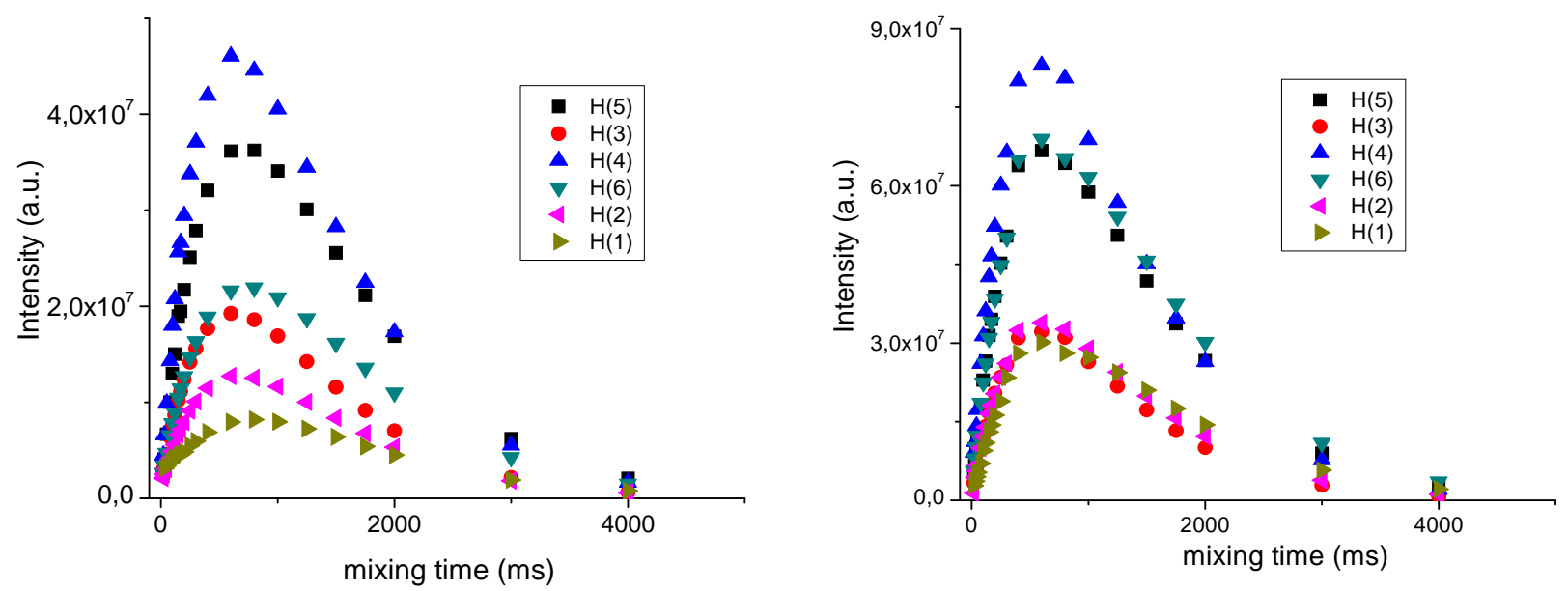

Figure 3. $\left\{{ }^{1} \mathrm{H}^{19}{ }^{\mathrm{F}}\right\}$ NOE build-up curves for PYR 13 TFSI (left) and $0.9 \mathrm{PYR}_{13} \mathrm{TFSI}-0.1$ LiTFSI (right). The cross-peak volumes are reported on the $y$ axis after normalization accounting for the total number of interacting nuclei (see Materials and Methods). The contact time for NOE development (the mixing time) is reported on the $x$ axis.
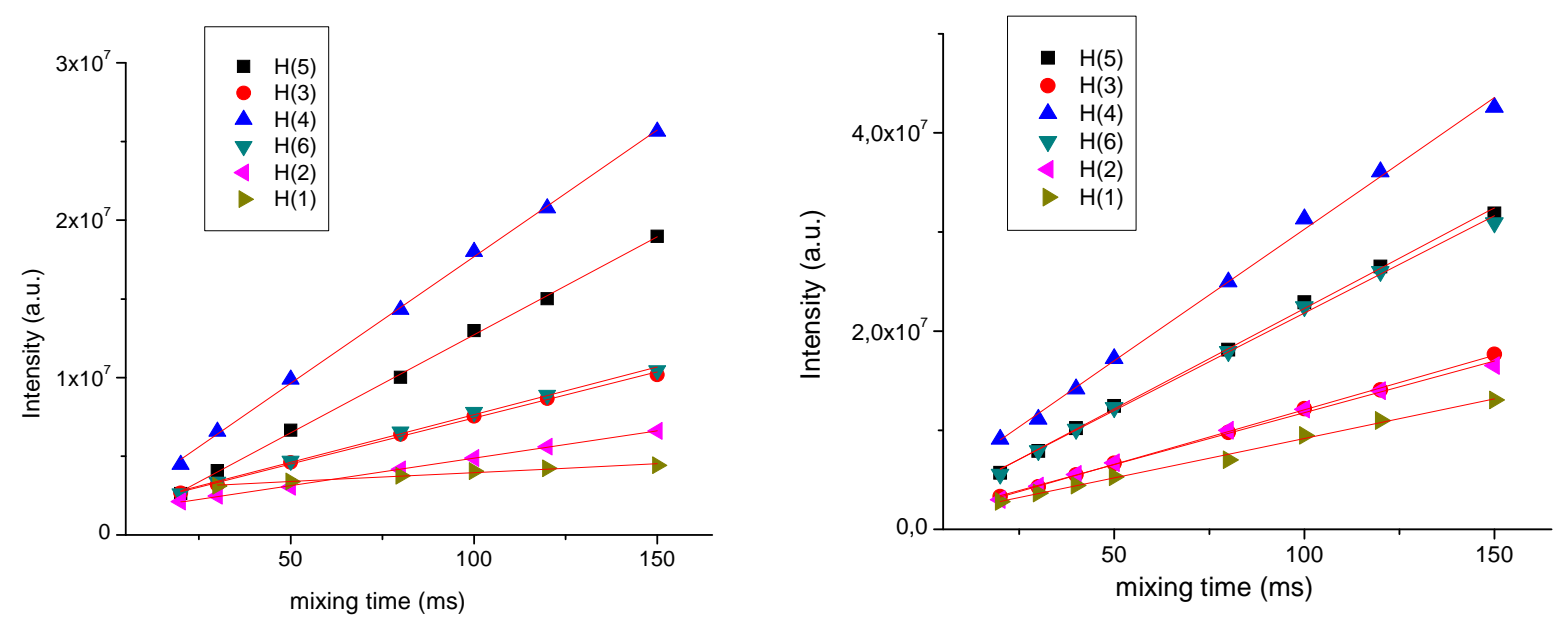

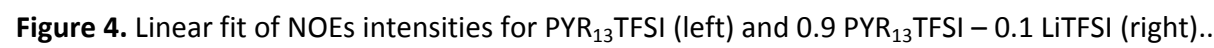

The normalized values of the NOE intensity are listed in Table 2. The arbitrary value 1 was given to the most intense interaction. All the others are scaled accordingly. 
Table 2. Normalized $\left\{{ }^{1} \mathrm{H}^{19}{ }^{19} \mathrm{~F}\right\}$ NOE intensity for pure $\mathrm{PYR}_{13} \mathrm{TFSI}$ and $\mathrm{PYR}_{13} \mathrm{FSI}$ and for their mixtures with LiTFSI. For clarity, also the code described in Table 1 is reported. The data of columns 2, 3 and 4 are referred to the NOE observed between the $\mathrm{CF}_{3}$ groups of TFSI with the $\mathrm{H}$ nuclei of the cation. In columns 5 and 6 are reported the NOE of the $\mathrm{CF}_{3}$ groups of TFSI and of the F nuclei of FSI with the $\mathrm{H}$ nuclei of the cation, respectively.

\begin{tabular}{|c|c|c|c|c|c|}
\hline \multirow[t]{2}{*}{ Protons } & \multicolumn{5}{|c|}{ Normalized $\left\{{ }^{1} \mathrm{H}^{19} \mathrm{~F}\right\}$ NOE } \\
\hline & $\begin{array}{l}\text { PYR }_{13} \text { TFSI } \\
\text { (PY1100) }\end{array}$ & $\begin{array}{l}\mathrm{PYR}_{13} \mathrm{FSI} \\
(\mathrm{PY} 1010)\end{array}$ & $\begin{array}{l}0.9 \text { PYR }_{13} \text { TFSI-0.1LiTFSI } \\
\text { (PY9101) }\end{array}$ & $\begin{array}{l}\text { 0.9PYR }_{13} \text { FSI-0.1 LiTFSI } \\
\text { (PY9191) }\end{array}$ & $\begin{array}{l}\text { 0.9PYR }_{13} \mathrm{FSI} 0.1 \text { LiTFSI }^{\mathrm{b}}{ }^{\mathrm{T}} \\
\text { (PY9191) }\end{array}$ \\
\hline$H(4)$ & 1 & 1 & 1 & 1 & 1 \\
\hline$H(5)$ & 0.77 & 0.80 & 0.76 & 0.85 & 0.95 \\
\hline$H(6)$ & 0.37 & 0.19 & 0.74 & 0.45 & 0.49 \\
\hline$H(3)$ & 0.36 & 0.2 & 0.41 & 0.25 & 0.29 \\
\hline$H(2)$ & 0.21 & 0.11 & 0.39 & 0.17 & 0.16 \\
\hline$H(1)$ & 0.07 & 0.13 & 0.30 & 0.00 & 0.00 \\
\hline
\end{tabular}

${ }^{\text {a }}$ Data referred to $\mathrm{PYR}_{13}$-TFSI NOE

${ }^{\mathrm{b}}$ Data referred to $\mathrm{PYR}_{13}-\mathrm{FSI} \mathrm{NOE}$

A more visual approach is proposed in Figure 5, where the molecular frame of the cation is wrapped in shaded elipsoids/spheres whose size is proportional to the intensity of the interaction. The colour code, red/blue for strong/medium-weak interaction, respectively, is also adopted. For clarity, the figures of Table 1 are also reported near the $\mathrm{H}$ atoms involved in the $\left\{{ }^{1} \mathrm{H}^{-19} \mathrm{~F}\right\}$ NOE. The shaded volumes of Figure 5 can be interpreted as "probability of presence" of the interacting spins. 


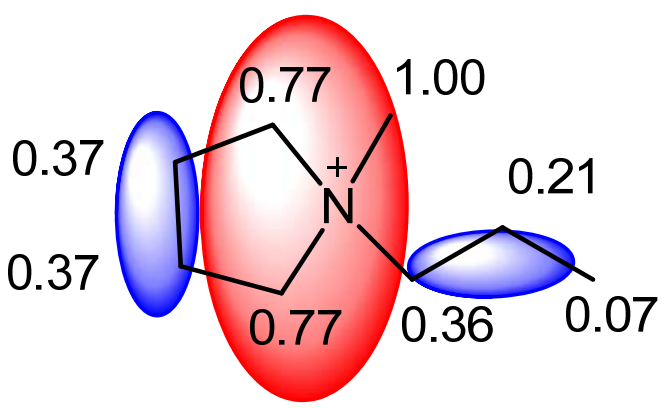

PYR $_{13}$ TFSI

(PY1100)

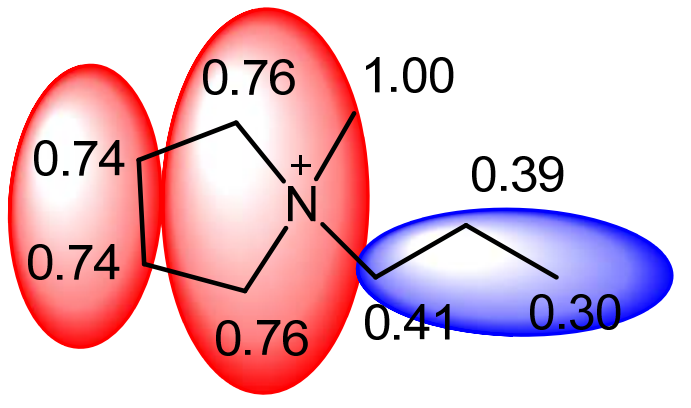

0.9 PYR $_{13}$ TFSI-0.1LITFSI

(PY9101)

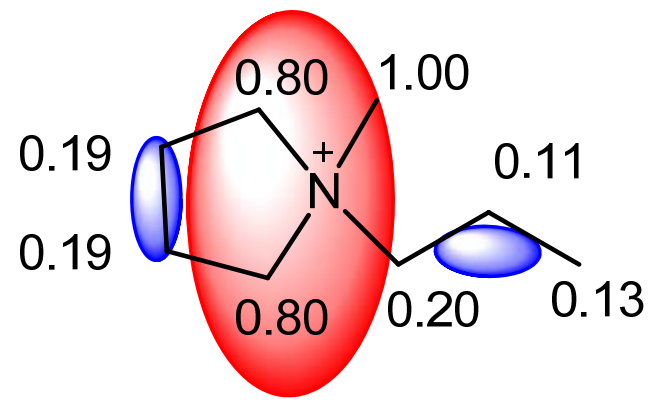

$\mathrm{PYR}_{13} \mathrm{FSI}$

(PY1010)

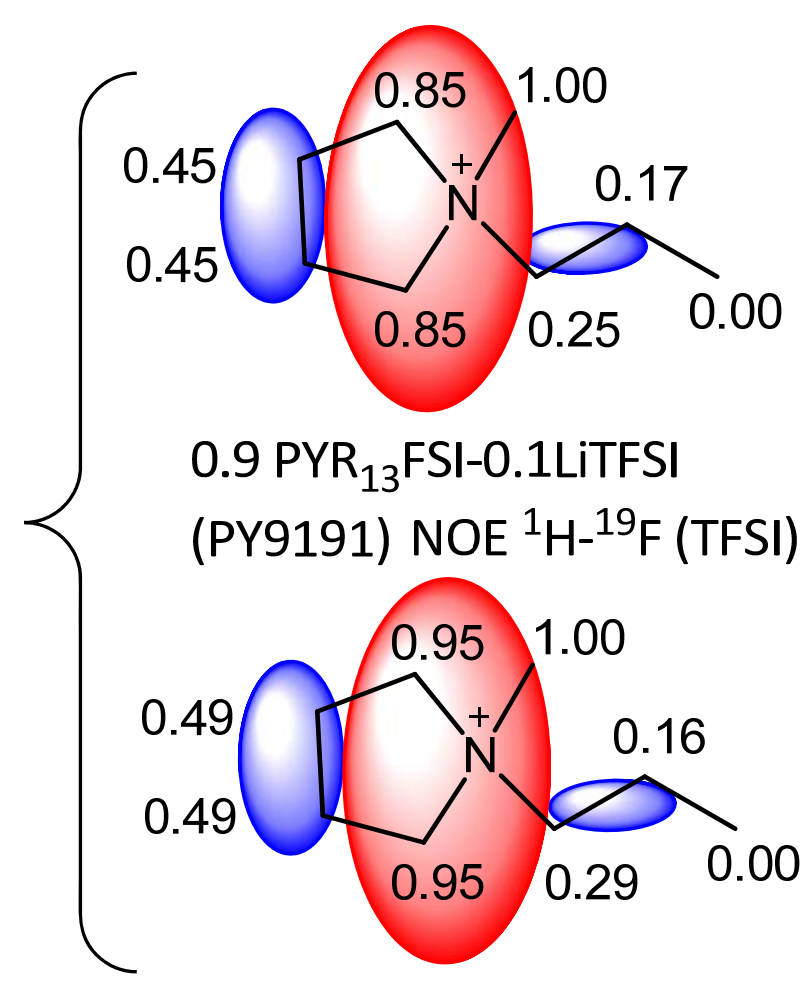

0.9 PYR $_{13}$ FSI-0.1LiTFSI
(PY9191) NOE ${ }^{1} \mathrm{H}^{19} \mathrm{~F}$ (FSI)

Figure 5. Graphical sketch of the normalized $\left\{{ }^{1} \mathrm{H}^{19}{ }^{19} \mathrm{~F}\right.$ NOE intensity for the pure ILs (left) and their mixtures with LiTFSI (right). The number are the NOE intensities taken from Table 1. The shaded volumes are proportional to the "probability of presence" of the fluorine atoms. The red colour indicated normalized intensity values greater than 0.5 . The NOE of the two different sets of ${ }^{19} \mathrm{~F}$ nuclei in the sample PY9191 (namely $\mathrm{CF}_{3}$ groups of TFSI and F of FSI) are shown in curly bracket from top to bottom, respectively.

According to the GSW theory, the $\left\{{ }^{1} \mathrm{H}-{ }^{19} \mathrm{~F}\right\} \mathrm{NOE}$ is expected to extend beyond the first solvation shell, due to the prevalence of the long-range effects over the short-range ones. The high and low value volumes are depicted in red and blue, and correspond to spatial regions either easily or not easily accessible to the $\mathrm{F}$ atoms of the anions, respectively. These results, and the proposed interpretation, outline the different domains in the IL rather than a localized, pair interaction of any individual cation with the closest anions. The concept of domain in ILs was initially proposed by Canongia-Lopes and Padua in 2006 from a 
theoretical viewpoint as a key feature of the nano-structuration in ILs [38]. More recently, experimental evidence have supported such a model $[39,40]$. The sketch reported in Figure 5 compare the extension of the anion accessibility domains for the ILs, PYR ${ }_{13} T F S I$ and PYR ${ }_{13} F S I$ with and without LiTFSI (the codes are: PY1100, PY1010, PY9101 and PY9191 in the order). Inspection of the sketches on the left hand side reveals quite clearly that the less polar part of the systems, namely the propyl chain and the $\mathrm{CH}_{2}-\mathrm{CH}_{2}$ part of the pyrrolidinium ring, account for the less probable presence of the anion. This point is in good agreement with the existence of a polar and non-polar domain within the liquids, and with the preferred presence of the anion in the polar domain. Noteworthy, the length of the alkyl substituent on the cations and the fluorine content on the anion are not high enough to provide evidence of the presence of fluorophilic domains, as previously reported for other ILs with broader apolar and fluorophilic components [40]. The smaller extension of the low probability area (blue elipsoids) in $\mathrm{PYR}_{13} \mathrm{FSI}$ compared to the corresponding in $\mathrm{PYR}_{13}$ TFSI is therefore ascribable to the different size of the anions.

It is interesting to observe if the addition of LiTFSI salt induces structural changes in the pure ionic liquids. The $\left\{{ }^{1} \mathrm{H}-{ }^{19} \mathrm{~F}\right\}$ NOE build-up curves for the LiTFSI doped samples, reported in Figure 4, show that the presence of the lithium salt strongly influences PYR ${ }_{13}$ TFSI. According to Figure 5 (top right), the main effect of doping PYR $_{13}$ TFSI with LiTFSI is to alter the size of the domain of anion accessibility, making it broader by extending the probability region to the whole pyrrolidinium ring, but scarcely influencing the propyl chain apolar domain. On the other hand, $\mathrm{PYR}_{13} \mathrm{FSI}$ does not show this effect in such a clear-cut way and when doped with LiTFSI salt.

\section{Lithium-proton HOESY}

NMR heteronuclear $\left\{{ }^{1} \mathrm{H}^{7} \mathrm{Li}\right\} \mathrm{NOE}$ correlation experiments were used to investigate the cation-lithium interactions in the pure ILs and their mixtures. It is known that the $\mathrm{Li}^{+}$ions are strongly complexed by sulfonilymide anions [41]. In a previous paper the X-ray structure of the $\left[\mathrm{Li}(\mathrm{TFSI}]_{2}\right]^{-}$ions was reported in pyrrolidinium type ILs with Li dopants.[42] The existence of tetra-coordinated Li ions is to be kept in mind when interpreting $\left\{{ }^{1} \mathrm{H}-{ }^{7} \mathrm{Li}\right\} \mathrm{NOE}$ data. An example of $\left\{{ }^{1} \mathrm{H}-{ }^{7} \mathrm{Li}\right\} \mathrm{NOE}$ build-up curve, along with the linear plot at short mixing time, is given in Figure 6. The normalized intensities are summarized in Table 3.
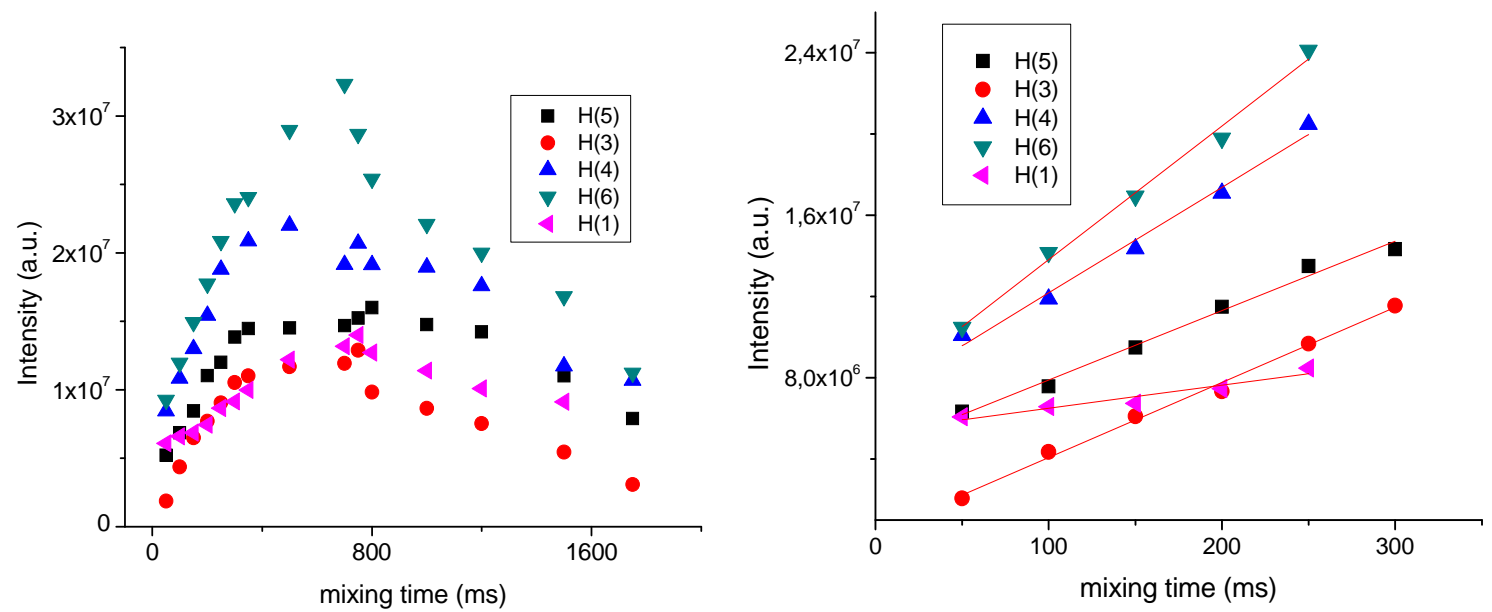

Figure 6. $\left\{{ }^{1} \mathrm{H}-{ }^{7} \mathrm{Li}\right\}$ NOE build up curves for 0.9PYR13TFSI-0.1LiTFSI and related linear fit. 
Table 3. Normalized $\left\{{ }^{1} \mathrm{H}^{7}{ }^{\mathrm{Li}}\right\} \mathrm{NOE}$ intensity for the mixtures 0.9 PYR $_{13}$ TFSI -0.1 LiTFSI (PY9101), 0.9 PYR ${ }_{13}$ FSI -0.1 LiTFSI (PY9191) and for the intermediate composition 0.3 PYR $_{13}$ TFSI-0.6 PYR 13 FSI- 0.1 LiTFSI (PY9461).

\begin{tabular}{|c|c|c|c|}
\hline \multirow[t]{2}{*}{ Protons } & \multicolumn{3}{|l|}{ Normalized $\left\{{ }^{1} H-{ }^{7} L i\right\} N O E$} \\
\hline & $\begin{array}{l}0.9 \text { PYR }_{13} \text { TFSI-0.1 LiTFSI } \\
\text { (PY9101) }\end{array}$ & $\begin{array}{l}0.3 \text { PYR }_{13} \text { TFSI-0.6 } \text { PYR }_{13} \text { FSI- } 0.1 \text { LiTFSI } \\
\text { (PY9461) }\end{array}$ & $\begin{array}{l}0.9 \text { PYR }_{13} F S I-0.1 \text { LiTFSI } \\
\text { (PY9191) }\end{array}$ \\
\hline$H(6)$ & 1 & 1 & 1 \\
\hline$H(4)$ & 0.79 & 0.65 & 0.52 \\
\hline$H(5)$ & 0.52 & 0.51 & 0.82 \\
\hline$H(3)$ & 0.56 & - & 0.14 \\
\hline$H(2)$ & - & - & 0.21 \\
\hline$H(1)$ & 0.17 & 0.63 & 0.93 \\
\hline
\end{tabular}

The sketch of the normalized $\left\{{ }^{1} \mathrm{H}-{ }^{7} \mathrm{Li}\right\}$ NOE intensities is displayed in Figure 7 , with the same settings as in Figure 5.

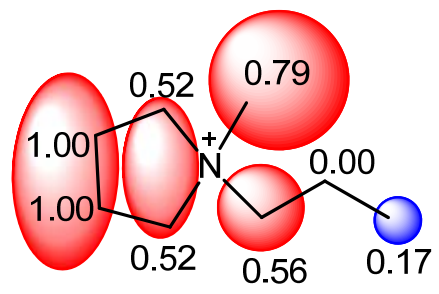

0.9 PYR $_{13}$ TFSI - 0.1 LiTFSI (PY9101)

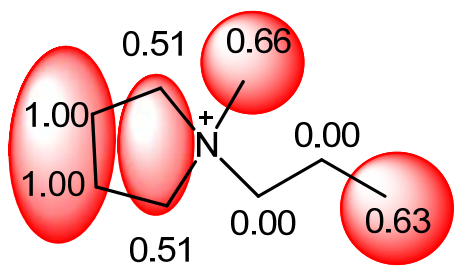

0.3 PYR ${ }_{13}$ TFSI - 0.6 PYR ${ }_{13}$ FSI - 0.1 LiTFSI (PY9461)

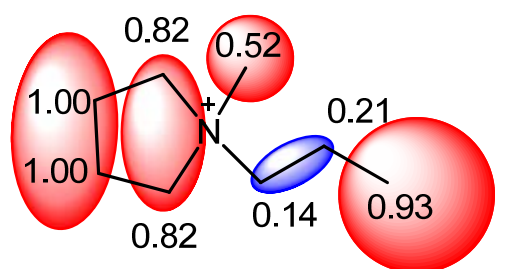

0.9 PYR $_{13} \mathrm{FSI}$ - 0.1 LiTFSI

(PY9191)

Figure 7. Graphical sketch of the normalized $\left\{{ }^{1} \mathrm{H}-{ }^{7} \mathrm{Li}\right\} \mathrm{NOE}$ intensity for the two mixtures $0.9 \mathrm{PYR}_{13} \mathrm{TFSI}-0.1 \mathrm{LiTFSI}$ (left, PY9101) and

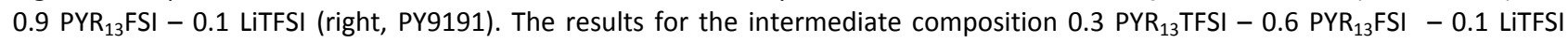
(PY9461, central panel) are also reported (see Discussion). The numbers are the NOE intensities taken from Table 3. The shaded volumes are proportional to the "probability of presence" of the lithium atoms. The red colour indicates normalized intensity values greater than 0.5 .

The data related to PY9101 (left panel) point out that the $\mathrm{Li}^{+}$ion, suitably chelated by TFSI ligands, is preferentially located around the positive charge of $\left[\mathrm{PYR}_{13}\right]^{+}$, consistent with the charge alternation pattern $\mathrm{PYR}_{13}{ }^{+} /\left[\mathrm{Li}(\mathrm{TFSI})_{2}\right]^{-}$. A possible involvement of $\mathrm{CF}_{3}{ }^{\cdots} \mathrm{H}_{3} \mathrm{C}-\mathrm{N}^{+}$hydrogen bonds, clearly observed in the solid state on DEME-TFSI based ILs [43], can be invoked to justify the high probability of presence of $\mathrm{Li}^{+}$in the proximity of the $\mathrm{N}$-methyl group of PY9101. Consistent with this interpretation is the fact that such probability decreases in PY9191 (Figure 7, right panel) as the hydrogen bond properties of [FSI] ${ }^{-}$are reported to be significantly lower than those of [TFSI] [43]. The pattern of $\left\{{ }^{1} \mathrm{H}-{ }^{7} \mathrm{Li}\right\} \mathrm{NOE}$ contacts of PY9101 can be also compared to the experimental H-Li non-bonded distances derived from the crystal structure of a similar system, namely 0.33 PYR $_{14}$ TFSI -0.67 LiTFSI. [42] The two systems are very similar but not identical: the alkyl chain is longer in the latter (C4 in the crystal structure vs C3 of the present study) and the IL/LiTFSI composition is also different ( $1 / 2$ in the crystal structure vs $9 / 1$ of the present study). Having considered these differences, the $\left\{{ }^{1} \mathrm{H}-{ }^{7} \mathrm{Li}\right\} N O E$ intensities are in a good qualitative agreement with the first neighbours $\mathrm{H}$ ' $\mathrm{Li}$ distances obtained from the X-ray data. A histogram of the crystal contacts is reported in Figure 8. 

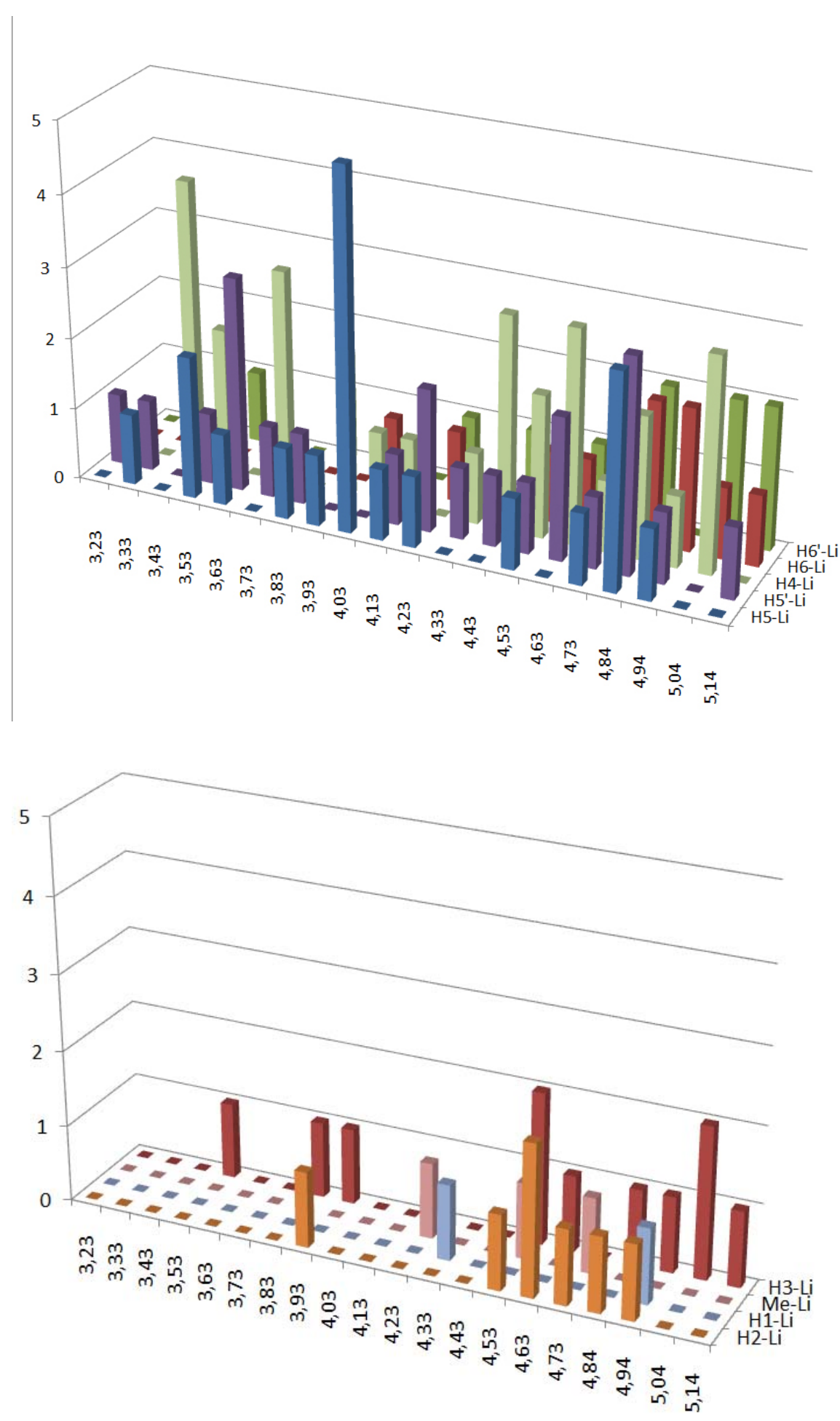

Figure 8. Histogram of frequencies for the $\mathrm{H}$...Li distances in the 3 to $5 \AA$ distance range for single crystal of the mixture 0.33 $\mathrm{PYR}_{14}$ TFSI -0.67 LiTFSI from ref. [42]. The $x$-axis shows the distance intervals (A) for the selected contacts. The $y$-axis shows the type of contact, according to the atom numbering of Figure 1 . The z-axis shows the bars' height: The z-axis scale is the integer number of counts for the selected contact in each distance interval. The top panel groups the $\mathrm{H}$ atoms on the PYR $\mathrm{R}_{14}$ ring (same numbering as in Figure 1: H5- $\mathrm{Li}, \mathrm{H} 5{ }^{\prime}-\mathrm{Li}, \mathrm{H} 4-\mathrm{Li}, \mathrm{H} 6-\mathrm{Li}$ and $\mathrm{H6}^{\prime}-\mathrm{Li}$ ), while the alkyl chain $\mathrm{H}$ atoms are separately analysed in the bottom panel (H2-Li, H1Li, Me-Li, H3-Li).

The category axis accounts for the H...Li distance intervals ( 3 to $5 \AA$ in $0.1 \AA$ steps), whereas the bars accounts for the number of contacts in each interval. The histogram reported on the left panel of Figure 8 clearly indicates that the frequency of short contacts in the range $3 \AA<r<5 \AA$ is high for the $H$ atoms in 
position 5,6 and for the $\mathrm{N}$-methyl group, thus mirroring the NOE intensities depicted in Figure 7 (left). In a parallel way, the right panel of Figure 8 points out that the alkyl chain is, on average, not in close proximity of the $\mathrm{Li}$ ion, again in nice qualitative agreement with the $\left\{{ }^{1} \mathrm{H}-{ }^{7} \mathrm{Li}\right\} \mathrm{NOE}$ data. From the viewpoint of this work, the fair agreement between the distance statistics derived from the single crystal structure and the $\left\{{ }^{1} \mathrm{H}-{ }^{7} \mathrm{Li}\right\} N O E$ patterns detected here supports the assumption that $\left\{{ }^{1} \mathrm{H}-{ }^{7} \mathrm{Li}\right\} N \mathrm{NOE}$ are mainly driven by the short-range interactions, as claimed in the Introduction and for the reason that the Larmor frequencies of the interacting nuclei are far apart. This point provides, on our opinion, an experimental support to the interpretation of intermolecular heteronuclear NOEs and it is in fair agreement with one of the key statement of the GSW theory: "frequency matters".

Finally, a comparison between the $\left\{{ }^{1} \mathrm{H}_{-}{ }^{7} \mathrm{Li}\right\}$ NOE patterns of PY9101 and PY9191 is worth of mention. Indeed, a striking feature arises from the comparison of the left and right panel of Figure 7. In the case of PY9191, a surprisingly high NOE intensity is detected for the methyl group of the propyl chain (H1), despite the evidence mentioned above of a scarce affinity of Li for the apolar tail of the PYR ${ }_{13}$ cation. This finding is completely counterintuitive and indeed represents a sort of conundrum. Further studies are in progress on a larger repertoire of compositions in order to work out a general interpretation. However, we would like to anticipate here just one result related to the mixture of composition $0.3 \mathrm{PYR}_{13} \mathrm{TFSI}-0.6 \mathrm{PYR}_{13} \mathrm{FSI}-0.1$ LiTFSI (PY9461, see Table 3) as an example of a representative intermediate composition between PY9101 and PY9191. The NOE data, depicted in the middle sketch of Figure 7, point out that the methyl group at the end of the propyl chain $(\mathrm{H} 1)$ is a highly sensitive probe to the anion composition of the mixture. The observed normalized intensites for the Li-H1 interaction are $0.17,0.63$ and 0.93 for PY9109, PY9461 and PY9191, in the order, thus suggesting that the interaction is modulated by the TFSI/FSI ratio. The latter, in turn, is seen to affect the steric hindrance of the coordinated $L i$ ions, with possible consequences on the structure and on the size of the first solvation shell of the Li ions. Additionally, the opposite trend is observed for the Li-H4 interaction: 0.79, 0.66, 0.52 for PY9109, PY9461 and PY9191, in the order. This trend seems to confirm the involvement of $\mathrm{CF}_{3}{ }^{\cdots} \mathrm{H}_{3} \mathrm{C}-\mathrm{N}^{+}$hydrogen bond capability, which intensity is, once again, modulated by the mole fraction of TFSI anon. These conclusions, although far from providing a general, clear-cut model for the Li solvation shell in these systems, are nevertheless consistent with the effect of short-range interactions monitored by ${ }^{1} \mathrm{H}^{-}{ }^{7} \mathrm{Li}$ NOE data.

\section{Conclusion}

The combined use of $\left\{{ }^{1} \mathrm{H}_{-}{ }^{19} \mathrm{~F}\right\}$ and $\left\{{ }^{1} \mathrm{H}_{-}{ }^{7} \mathrm{Li}\right\} \mathrm{HOESY}$ experiments allowed us to observe the same molecular panorama from two different observation sites. The distance dependence of the heteronuclear, intermolecular NOE on the Larmor frequencies of the interacting nuclei can be exploited to gain structural information concerning different distance ranges. The experimental approach here described, and the data analysis considering the GSW theory, provide structural details on both the short-range local structure of anion-cation alternation pattern and the long-range aggregation motives generating the different domains in ILs. Overall, the method and data analysis here proposed represent a paradigmatic example of the rethinking of structural assessment of the local order in ILs derived by intermolecular NOE data.

As a final remark, we hope to stimulate experimentalists to a renewed way of interpreting NMR data and the theoretician to use the experimental approach and results here described as a suitable input for MD simulation, for the validation of the intermolecular NOE models and to work out quantitative indication about the influence of the first solvation shells upon the intermolecular NOE patterns, especially for the $\left\{{ }^{1} \mathrm{H}-{ }^{1} \mathrm{H}\right\}$ and $\left\{{ }^{1} \mathrm{H}-{ }^{19} \mathrm{~F}\right\}$ experiments, by far the most used and useful for ILs.

Acknowledgments. The authors acknowledge the EU for funding (FP7 - GREENLION Project - Contract $n$ ㅇ 285268). AM thanks EU COST Action CM1206 EXIL (Exchange in Ionic Liquids). The authors thank Dr Alessandro Triolo (CNR - Italy) for the fruitful discussion.

\section{References}

[1] A.-L. Rollet, C. Bessada, NMR studies of molten salt and room temperature ionic liquids, Annu. Rep. NMR Spectrosc. 78 (2013) 143-207. 
[2] H. Weingärtner, NMR studies of molten salt and room temperature ionic liquids, Curr. Opin. Colloid Interface Sci. 18 (2013) 183-189.

[3] D. Neuhaus, M. P. Williamson, The Nuclear Overhauser Effect in Structural and Conformational Analysis, second ed., Wiley-VCH. Weinheim, 2000.

[4] A. Bagno, D. Rastrelli, G. Saielli, NMR techniques for the investigation of solvation phenomena and non-covalent interactions, Prog. Nucl. Magn. Reson. Spectrosc. 47 (2005) 41-93.

[5] R. A. Mantz, P. C. Trulove, R. T. Carlin, R. A. Ostryoung, ROESY NMR of basic ambient-temperature chloroaluminate ionic liquids, Inorg. Chem. 34 (1995) 3846-3847.

[6] A. Mele, C. D. Tran, S. H. De Paoli Lacerda, The structure of a room-temperature ionic liquid with and without trace amounts of water: the role of $\mathrm{C}-\mathrm{H} \cdots \mathrm{O}$ and $\mathrm{C}-\mathrm{H} \cdots \mathrm{F}$ interactions in 1-n-butyl-3methylimidazolium tetrafluoroborate, Angew. Chem. Int. Ed. 42 (2003) 4364-4366.

[7] A. Mele, G. Romanò, M. Giannone, E. Ragg, G. Fronza, G. Raos, V. Marcon, The local structure of

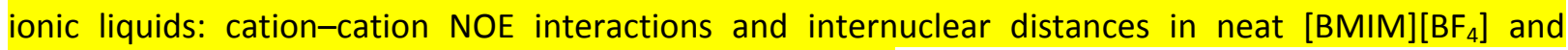
[BDMIM] [BF 4 ], Angew. Chem. Int. Ed. 45 (2006) 1123-1126

[8] T. Gutel, C. C. Santini, A. A. H. Pádua, B. Fenet, Y. Bernard; J. N. Canongia Lopes, F, Bayard, M. F. Costa Gomes, A. S. Pensado, Interaction between the $\pi$-system of toluene and the imidazolium ring of ionic liquids: A combined NMR and molecular simulation study, J. Phys. Chem. B 113 (2009) 170-177.

[9] S. Puttick, A. L. Davis, K. Butler, L. Lambert, J. El Harfi, D. J. Irvine, A. K. Whittaker, K. J. Thurecht, P. Licence, NMR as a probe of nanostructured domains in ionic liquids: Does domain segregation explain increased performance of free radical polymerization? Chem. Sci. 2 (2011) 1810-1816.

[10] S. Khatun, E. W. Castner, lonic liquid-solute interactions studied by 2D NOE NMR spectroscopy, J. Phys. Chem. B doi: 0.1021/jp509861g.

[11] F. Castiglione, M. Moreno, G. Raos, A. Famulari, A. Mele , G. B. Appetecchi, S. Passerini, Structural organization and transport properties of novel pyrrolidinium-based ionic liquids with perfluoroalkyl sulfonylimide anions, J. Phys. Chem. B 113 (2009) 10750-10759.]

[12] Y. Lingscheid, S. Arenz, R. Giernoth, Heteronuclear NOE spectroscopy in ionic liquids, Chem. Phys. Chem. 13 (2012) 261-266.

[13] B. Halle, Cross-relaxation between macromolecular and solvent spins: The role of long-range dipole couplings, J. Chem. Phys. 119 (2003) 12372-12385.

[14] D. Frezzato, F. Rastrelli, A. Bagno, Nuclear spin relaxation driven by intermolecular dipolar interactions: The role of solute-solvent pair correlations in the modeling of spectral density functions, J. Phys. Chem. B 110 (2006) 5676-5689.

[15] A. Mele, NOE experiments for ionic liquids: Tools and strategies, Chimica Oggi/Chemistry Today 28 (2010) 48-55.

[16] S. Gabl, O. Steinhauser, H. Weingärtner, From short-range to long-range intermolecular NOEs in ionic liquids: Frequency does matter, Angew. Chem. Int. Ed. 52 (2013) 9242-9247.

[17]. S. Gabl, C. Schröder, D. Braun, H. Weingärtner, O. Steinhauser, Pair dynamics and the intermolecular nuclear Overhauser effect (NOE) in liquids analysed by simulation and model theories: Application to an ionic liquid, J. Chem. Phys. 140 (2014) 184503 1-17.

[18] F. Castiglione, A. Famulari, G. Raos, S. V. Meille, A. Mele, G. B. Appetecchi, S. Passerini, Pyrrolidinium-based ionic liquids doped with lithium salts: How does $\mathrm{Li}^{+}$coordination affect Its diffusivity?, J. Phys. Chem. B 118 (2014) 13679-13688.

[19] G.B. Appetecchi, M. Montanino, S. Passerini, lonic liquid-based electrolytes for high-energy lithium batteries, in: A.E. Vissser, N.J. Bridges, and R.D. Rogers (Eds), ACS Symposium Series 1117, Oxford University Press, Inc., American Chemical Society, Washington, DC, USA, 2013.

[20] Z.-B. Zhou, H. Matsumoto, K. Tatsumi, Low-melting, low-viscous, hydrophobic ionic liquids: Aliphatic quaternary ammonium salts with perfluoroalkyltrifluoroborates, Chem. Eur. J. 11 (2005) 752766.

[21] W. Xu, E. Cooper, A. A. Angell, lonic liquids: ion mobilities, glass temperatures, and fragilities, J. Phys. Chem. B 107 (2003) 6170-6178.

[22] J.R.D. Rogers, K.R. Seddon (Eds), lonic liquids: Industrial application to green chemistry, ACS Symposium Series 818, Oxford University Press, Inc., American Chemical Society, Washington, DC, USA, 2002. 
[23] H. Ohno (Ed), Electrochemical aspects of ionic liquids, John Wiley \& Sons Inc., Hoboken, New Jersey, 2005.

[24] A.I. Bhatt, I. May, V.A. Volkovich, M.E. Hetherington, B. Lewin, R.C. Thied, N.J. Ertok, Group 15 quaternary alkyl bistriflimides: ionic liquids with potential application in electropositive metal deposition and as supporting electrolytes, Chem. Soc. Dalton Trans. (2002) 4532-4534.

[25] S. Panozzo, M. Armand, O. Stephan, Light-emitting electrochemical cells using a molten delocalized salt, Appl. Phys. Lett. 80 (2002) 679-683.

[26] P. Wang, S.M. Zakeeruddin, I. Exnar, M. Gratzel, High efficiency dye-sensitized nanocrystalline solar cells based on ionic liquid polymer gel electrolyte, Chem. Commun. 2002, 2972-2973.

[27] J. Fuller, A.C. Breda, R.T. Carlin, Ionic liquid-polymer gel electrolytes from hydrophilic and hydrophobic ionic liquids, Electroanal. Chem. 459 (1998) 29-34.

[28] H. Nakagawa, S. Izuchi, K. Kunawa, T. Nukuda, Y. Aihara, Liquid and polymer gel electrolytes for lithium batteries composed of room-temperature molten salt doped by lithium salt, J. Electrochem. Soc. 150 (2003) A695-A700.

[29] A. Noda, M.A.B.H. Susan, K. Kudo, S. Mitsushima, K. Hayamizu, M. Watanabe, Brønsted acid-base ionic liquids as proton-conducting nonaqueous electrolytes, J.Phys. Chem. B 107 (2003) 4024-4033.

[30] A. Balducci, W.A. Henderson, M. Mastragostino, S. Passerini, P. Simon, F. Soavi, Cycling stability of a hybrid activated carbon//poly(3-methylthiophene) supercapacitor with N-butyl-Nmethylpyrrolidinium bis(trifluoromethanesulfonyl)imide ionic liquid as electrolyte, Electrochim. Acta 50 (2005) 2233-2237.

[31] W.A. Henderson, S. Passerini, Phase behavior of ionic liquid-LiX mixtures: Pyrrolidinium cations and TFSl anions, Chem. Mater. 16 (2004) 2881-2885.

[32] F. Castiglione, E. Ragg, A. Mele, G. B. Appetecchi, M. Montanino, S. Passerini, Molecular environment and enhanced diffusivity of $\mathrm{Li}^{+}$ions in lithium-salt-doped ionic liquid electrolytes, J. Phys. Chem. Letters 2 (2011) 153-157.

[33] Y.-J. Lin, L. Hao, Y.-C. Lin, C.-W. Kuo, P.-R. Chen, T.-Y Wu, I.-W. Sun, A comparative study of ionic conductivity, translational diffusion, molecular motion, and physicochemical properties in lithium bis(trifluoromethanesulfonyl)imide-doped 1-methyl-3-pentyl- and 1,2-dimethyl-3-pentyl-substituted imidazolium-based ionic liquids, Int. J. Electrochem. Sci. 8 (2013) 8097-8114.

[34 J.-P. Baltaze, P. Judeinstein, Probing ion coordination in polymer electrolytes with multinuclear NMR correlation spectroscopy, Solid State lonics 181 (2010) 672-677.

[35] M. Montanino, F. Alessandrini, S. Passerini, G.B. Appetecchi, Water-based synthesis of hydrophobic ionic liquids for high-energy electrochemical devices, Electrochim. Acta, 96 (2013) 124-133.

[36] T. M. Alam, D. M. Pedrotty, T. J. Boyle, Modified, pulse field gradient-enhanced inverse-detected HOESY pulse sequence for reduction of $t 1$ spectral artifacts, Magn. Reson. Chem. 40 (2002) 361-365.

[37] S.-S. Hou, J.-K. Tzeng and M.-H. Chuang, Intermolecular association and supramolecular structures of PNVF-LiPFN and PVP-LiPFN complexes in the aqueous phase, Soft Matter 6 (2010) 409-415.

[38] J. N. A. Canongia Lopes, A. A. H. Pádua, Nanostructural organization in ionic liquids, J. Phys. Chem. B 110 (2006) 3330-3335.

[39] A. Triolo, O. Russina, H.-J. Bleif, E. Di Cola Nanoscale segregation in room temperature ionic liquids, J. Phys. Chem. B 111 (2007) 4641-4644.

[40] O. Russina, F. Lo Celso, M. Di Michiel, S. Passerini, G. B. Appetecchi, F. Castiglione, A. Mele, R. Caminiti, A. Triolo, Mesoscopic structural organization in triphilic room temperature ionic liquids, Faraday Discuss. 167 (2013) 499-513.

[41] A. Martinelli, A. Matic, P. Jacobsson, L. Beorjesson, A. Fernicola, B. Scrosati, Behavior and ionic conductivity in lithium bis(trifluoromethanesulfonyl)imide-doped ionic liquids of the pyrrolidinium cation and bis(trifluoromethanesulfonyl)imide anion, J. Phys. Chem. B 113 (2009) 11247-11251.

[42] Q. Zhou, P. D. Boyle, L. Malpezzi, A. Mele, J.-H. Shin, S. Passerini, W. A. Henderson, Phase behavior of ionic liquid-LiX mixtures: Pyrrolidinium cations and TFSl'anions - Linking structure to transport properties, Chem. Mater 23 (2011) 4331-4337.

[43] Y. Yoshida, G. Saito,lonic liquid based on diethylmethyl(2-methoxyethyl)ammonium cations and bis(perfluoroalkanesulfonyl)amide anions: Influence of anion structure on liquid properties, Phys. Chem. Chem. Phys. 13 (2011) 20302-20310. 
Graphical Abstract

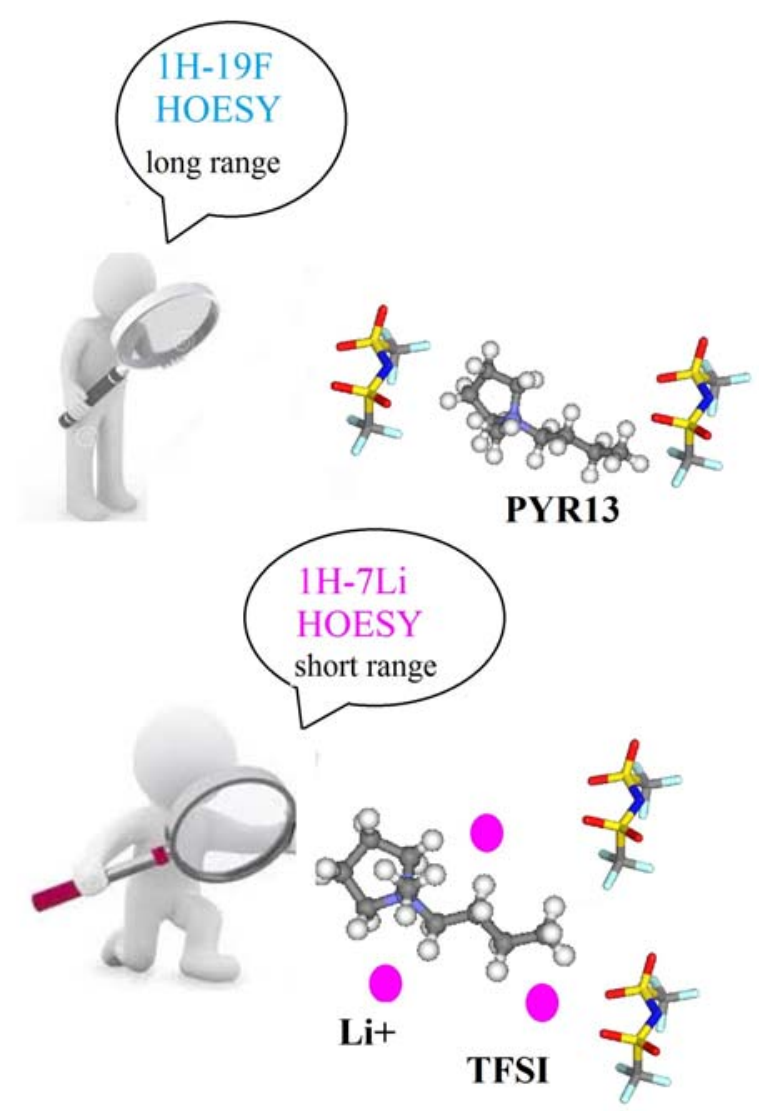

